\title{
CFTR 5T variant has a low penetrance in females that is partially attributable to its haplotype
}

Weimin Sun, $P h D^{1}$, Ben Anderson, BS ${ }^{1}$, Joy Redman, $M S^{1}$, Aubrey Milunsky, DSc ${ }^{3}$, Arlene Buller, $P h D^{1}$, Matthew J. McGinniss, $P h D^{1}$, Franklin Quan, $P h D^{1}$, Arturo Anguiano, $M D^{2}$, Stephen Huang, $P h D^{1}$, Feras Hantash, $P h D^{1}$, and Charles Strom, $M D, P h D^{1}$

\begin{abstract}
Purpose: The study's purpose was to understand the molecular basis for different clinical phenotypes of the 5T variant, a tract of 5 thymidines in intron 8 of the cystic fibrosis transmembrane conductance regulator (CFTR) gene, which disrupts processing of CFTR mRNA and reduces synthesis from the corresponding CFTR alleles. Method: We analyzed the polymorphic TG dinucleotide repeat adjacent to the 5T variant in intron 8 and the codon 470 in exon 10. Patients selected for this study were positive for both the $5 \mathrm{~T}$ variant and the major cystic fibrosis mutation, Delta F508. Almost all Delta F508 mutation alleles occur in a 10TG-9T-470M haplotype. Therefore, it is possible to determine the haplotype of the 5T variant in trans. Results: Of the 74 samples analyzed, 41 (55\%) were 11TG-5T-470M, 31 (42\%) were 12TG-5T-470V, and 2 (3\%) were 13TG-5T-470M. Of the 49 cases for which we had clinical information, $17.6 \%$ of females (6/34) and $66.7 \%$ of males (10/15) showed symptoms resembling atypical cystic fibrosis. The haplotype with the highest penetrance in females (42\% or 5/12) and more than 80\% (5/6) in males is 12TG-5T-470V. We also evaluated 12 males affected with congenital bilateral absence of vas deferens and positive for the 5T variant; 10 of 12 had the 12TG-5T-470V haplotype. Conclusion: Overall, the 5T variant has a milder clinical consequence than previously estimated in females. The clinical presentations of the 5T variant are associated with the 5T-12TG-470M haplotype. Genet Med 2006:8(6):339-345.
\end{abstract}

Key Words: cystic fibrosis, $5 T$ variant, haplotype, penetrance, CBAVD

Cystic fibrosis (CF) is the most common life-limiting recessive genetic disease in whites. ${ }^{1,2}$ Mutations in the cystic fibrosis transmembrane conductance regulator (CFTR) gene are responsible for the disease in almost all patients with CF. ${ }^{3,4}$ More than 1000 mutations and variants in the CFTR gene have been detected in patients with CF and families associated with the disease. ${ }^{5}$ CFTR mutations can lead to a wide spectrum of phenotypes ranging from severe pulmonary disease with pancreatic insufficiency to atypical presentations such as chronic idiopathic pancreatitis, ${ }^{6,7}$ male infertility caused by congenital bilateral absence of vas deferens (CBAVD), ${ }^{8,9}$ or respiratory system conditions including chronic rhinosinusitis, ${ }^{10,11}$ bronchiectasis, ${ }^{12}$ and allergic bronchopulmonary aspergillosis. ${ }^{13,14}$ In addition, mild CF mutations may have reduced penetrance or produce different clinical phenotypes, dependent on the genetic background of the chromosome on which the mutation occurs. ${ }^{15,16}$

\footnotetext{
From the Departments of ${ }^{1}$ Molecular Genetics and ${ }^{2}$ Cytogenetics, Quest Diagnostics Nichols Institute, San Juan Capistrano, California, and ${ }^{3}$ Center for Human Genetics, Boston University School of Medicine, Boston, Massachusetts.

Weimin Sun, PhD, Department of Molecular Genetics Quest Diagnostics Nichols Institute, 33608 Ortega Highway, San Juan Capistrano, CA 92690.

Submitted for publication October 9, 2005.

Accepted for publication March 2, 2006.
}

DOI: 10.1097/01.gim.0000223549.57443.16
A polymorphic string of thymidines exists near the splice acceptor site in intron 8 of the CFTR gene. Among the three different major variants (5T, 7T, and 9T), the 5T allele is associated with the most inefficient use of the nearby splice acceptor site. ${ }^{17}$ This leads to a large proportion of the CFTR transcript missing exon 9, which codes for a part of the important first nucleotide-binding domain. ${ }^{18}$ An exon 9 negative CFTR protein will not mature and function as a chloride channel on apical cell membrane. ${ }^{19,20}$ Increased frequency of the 5T allele has been described in various atypical CFTR-associated diseases, including CBAVD, ${ }^{16,21}$ chronic idiopathic pancreatitis, ${ }^{6,7}$ and disseminated bronchiectasis. ${ }^{22}$ The $5 \mathrm{~T}$ allele, however, is also commonly seen in the general population. We observed an allelic frequency of 0.04 in approximately 320,000 individuals who underwent population-based CF carrier screening in our laboratory. ${ }^{23}$ Of the individuals positive for the $5 \mathrm{~T}$ allele, $2.42 \%(601 / 24,807)$ were also heterozygous for a classic CF mutation in our test population (unpublished data). The $5 \mathrm{~T}$ allele is believed to have reduced penetrance because individuals positive for both the $5 \mathrm{~T}$ allele and a classic CF mutation may or may not be symptomatic.

The same study from our group with more than 300,000 CF mutation screening cases showed that the $5 \mathrm{~T}$ variant has much lower penetrance than the previously estimated 0.5 or 0.6 . In patients compound heterozygous for the $\Delta \mathrm{F} 508$ mutation and the $5 \mathrm{~T}$ variant, only $3.8 \%$ of females $(7 / 184)$ and $34 \%$ of males 
(12/35) presented with symptoms resembling atypical CFTR diseases. ${ }^{23}$

Efforts have been made to understand potential modifying factors for the 5T allele. Cuppens et al. ${ }^{24}$ studied the possible effects of a variable tract of TG repeats in intron 8 (IVS-8) and the M470V polymorphism in exon 10 in modifying the splicing efficiency of the poly $\mathrm{T}$ alleles for exon 9 . On a 7T background, CFTR genes carrying 12TG repeats have six times as many transcripts lacking exon 9 compared with those with 10TG. Other studies also showed that 12TG-5T alleles had a higher representation in individuals affected with mild CFTRassociated diseases such as CBAVD, ${ }^{16}$ asthma, ${ }^{22}$ or chronic pancreatitis ${ }^{25}$ than in healthy controls. The M470V polymorphism in exon 10 of the CFTR gene has also been reported to influence intrinsic chloride channel activity of the CFTR protein in an in vitro study. ${ }^{24}$ In these studies, haplotypes for the $5 \mathrm{~T}$ alleles were determined by segregation studies in patients' families or were derived from known $5 \mathrm{~T}$ haplotypes. Because it would be difficult to accurately evaluate the penetrance of a $5 \mathrm{~T}$ allele when a weak CF mutation or no mutation is detected in trans, we only included individuals who are heterozygous for a classic disease-causing CF mutation, $\Delta \mathrm{F} 508$, and compound heterozygous for the $5 \mathrm{~T}$ and $9 \mathrm{~T}$ alleles. We evaluated the overall penetrance of $5 \mathrm{~T}$ alleles in relation to its chromosomal background, namely, the adjacent TG repeats in intron 8 and the M470V (1540 A>G) polymorphism in exon 10.

\section{MATERIALS AND METHODS}

\section{Patients}

Samples from the common CF mutation-screening assay and a comprehensive CFTR gene sequence analysis that tested positive for the $\Delta \mathrm{F} 508$ mutation and the $5 \mathrm{~T}$ variant were included in this study. When available, test indication and clinical phenotype information provided by physicians' offices were used. The samples were de-identified to ensure patient confidentiality. Genomic DNA samples from an additional eight male patients affected with CBAVD and positive for the 5T variant were part of previous studies. ${ }^{26,27}$

\section{DNA isolation}

Genomic DNA material was prepared from peripheral blood specimens submitted for CFTR gene mutation analysis on a 9604 BioRobot or a M96 BioRobot (Qiagen, Inc., Venlo, The Netherlands) as described previously. ${ }^{23}$ The average concentration of an extracted DNA sample was 15 to $50 \mathrm{ng} / \mu \mathrm{L}$.

\section{Common CFTR gene mutation detection}

Genomic DNA samples were subjected to multiplex amplification of CFTR gene regions and mutation detection by oligonucleotide ligation assay using reagents supplied by Abbott Diagnostics (Abbott Park, IL) as described previously. ${ }^{23}$ Polymerase chain reaction-oligonucleotide ligation assay products were analyzed on an ABI3100 automated DNA sequencer, and data analysis was performed using the GeneMapper software (Applied Biosystems, Foster City, CA). Thirty-two CF mutations including the 25 mutations recommended by the American College of Medical Genetics/American College of Obstetricians and Gynecologists (ACMG/ACOG) are detected.

\section{Direct sequence analysis of CFTR gene segments}

For the majority of the samples in this study, the segments amplified included part of intron 8 (containing the poly-T track and TG repeat region) exon 9 and exon 10. In cases in which comprehensive sequence analyses were performed to detect $\mathrm{CF}$ mutations, 32 fragments including all 27 exons of the CFTR gene and their splice junction sites, part of the promoter, and two intronic fragments (i11 and i19) were amplified as described previously. ${ }^{28}$

The CFTR gene segments were each amplified in individual polymerase chain reactions using primers with M13 linkers. Amplified products were subjected to a digestion with exonuclease I and calf intestinal phosphatase. Cycle-sequencing reactions for both strands of individual segments were then performed using the BigDye 3.1 reagents (Applied Biosystems) with M13 linker primers ( $5^{\prime}$ or $\left.3^{\prime}\right)$. Products from the cyclesequencing reactions were processed using calf intestinal phosphatase and analyzed on an ABI3730 automated DNA sequencer (Applied Biosystems). Sequencing data were stored in a BioLIMS database and analyzed using the SeqScape software (Applied Biosystems).

\section{RESULTS}

\section{T allele is the most frequently detected CF variant}

Population-based carrier screening using a 32-mutation reagent system (including the ACMG/ACOG-recommended 25mutation core panel) has been offered at Quest Diagnostics since 2001. In our previous report of the first 20,000 samples analyzed, allelic frequency of the $5 \mathrm{~T}$ allele was $4.6 \%$ (1846/ 40,206 chromosomes). In a follow-up study with a much larger dataset of more than 330,000 screening specimens (Table 1), the allelic frequency of the $5 \mathrm{~T}$ allele was $4.2 \%$. The allelic fre-

Table 1

The $5 \mathrm{~T}$ variant is frequently detected in our test population

\begin{tabular}{lr}
\hline PolyT status & Cases \\
\hline $5 \mathrm{~T} / 5 \mathrm{~T}$ & 975 \\
$5 \mathrm{~T} / 7 \mathrm{~T}$ & 23,922 \\
$5 \mathrm{~T} / 9 \mathrm{~T}$ & 3521 \\
$7 \mathrm{~T} / 7 \mathrm{~T}$ & 255,642 \\
7T/9T & 66,518 \\
9T/9T & 4875 \\
Total & 355,453 \\
5T population frequency & 0.080 \\
5T allelic frequency & 0.041 \\
\hline
\end{tabular}


quency of the $5 \mathrm{~T}$ variant is higher than the collective frequency of all the CF mutations detected in our test population, which is $3.03 \%(1 / 33)$. In addition, among the individuals positive for at least one $5 \mathrm{~T}$ variant, $2.42 \%(601 / 24807)$ of them were also positive for a CF mutation. Therefore, 1 in 591 individuals $(601 / 355,453)$ in our test population was positive for both the $5 \mathrm{~T}$ variant and a common CF mutation. The overrepresentation of the $5 \mathrm{~T}$ variant in the CF carrier screening population may be attributable to its reduced penetrance as in the case of other mild CF mutations or variants. ${ }^{29}$

\section{Specimens compound heterozygous for the $\Delta F 508$ mutation and the $5 \mathrm{~T}$ variant are chosen for this study}

$\Delta \mathrm{F} 508$ is the most prevalent mutation in CF chromosomes. Because it is a relatively ancient CF mutation, more than $95 \%$ of the $\Delta \mathrm{F} 508$ mutation alleles occur on a haplotype that includes 9T in intron 8. As shown in Figure 1, sequence analysis of a randomly chosen patient sample homozygous for the $\Delta$ F508 mutation indicated that the $\Delta$ F508 haplotype is generally found with $10 \mathrm{TG}$ at the (TG)n locus and 9T at the polyT locus in intron 8. It was also found to be associated with $1540 \mathrm{~A}$ (M470) in exon 10.

In the current study, we selected samples heterozygous positive for both the $\Delta$ F508 mutation and the $5 \mathrm{~T}$ variant. The presence of additional mutations were ruled out in 15 specimens by direct sequence analysis of all the coding exons and their corresponding splice junction sites, as well as the promoter region of the CFTR gene. Presence of deletion or duplication mutations involving one or more of the CF gene exons have been ruled out in selected samples using a method described elsewhere. ${ }^{31}$ In the remainder of the samples, only the presence of an additional 31 common CF mutations was ruled out. These mutations include R347H, S549N, S549R,
3876delA, 394delT, 3905insT, and V520F, in addition to the 25-mutation core panel recommended by ACMG/ACOG for population-based CF screening.

\section{T variant has three different haplotypes in our test samples}

A total of 74 patient specimens compound heterozygous for the $\Delta \mathrm{F} 508$ mutation and the $5 \mathrm{~T}$ variant were included in this study; 59 of them ( 47 females and 12 males) were selected from patients who underwent our common CF mutation-screening assay. An additional 15 patients (5 females and 10 males) were selected from specimens submitted for comprehensive CFTR gene sequence analysis based on their genotype results (compound heterozygous for the $\Delta \mathrm{F} 508$ mutation and the $5 \mathrm{~T}$ variant). From the specimens identified from the CF mutationscreening assay, two CFTR gene regions corresponding to intron 8-exon 9 and exon 10 were amplified and subjected to cycle-sequencing analysis. The presence of the $\Delta$ F508 mutation and the 5T variant was confirmed in all samples. In addition, none of them showed heterozygosity for the 7T allele (which is infrequently observed in $\Delta \mathrm{F} 508$ samples), whereas a heterozygosity for 9T-10TG in IVS-8 was detected in all samples. All samples in the study were also positive for at least one copy of the 1540A (M470) polymorphism in exon 10. These results indicate that there was probably no exception to the common haplotype on which the $\Delta$ F508 mutation is found.

As shown in Figure 2, the 5T variant in our study samples has three different haplotypes: 5T-11TG-M470, 5T-12TGV470, and 5T-13TG-M470. The 5T-11TG-M470 haplotype is the most prevalent haplotype among the three, accounting for $57 \%(41 / 74)$ of the 5 T alleles in this study (Table 2). We did not observe two other haplotypes the $5 \mathrm{~T}$ variant had been reported to have in previous studies, 5T-11TG-V470 and 5T-13TG-V470,22,25 likely because the sampling size of our study was small.

\section{TG (n)} polyT

$\Gamma$

tgatgtgtgtgtgtgtgtgtgtgtgt t t t t a a

$\ldots \ldots \ldots \ldots \ldots \ldots \ldots$

TGATGTGTGTGTGTGTGTGTGTGTTTT T T T T T A A

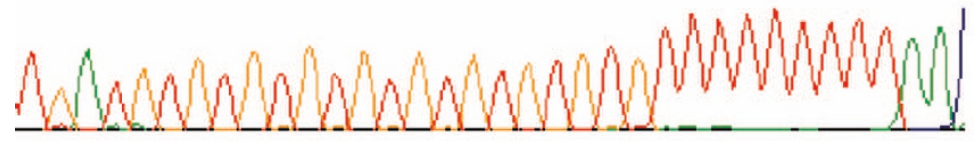

TGATGTGTGTGTGTGTGTGTGTGTTTTTTTTTA A

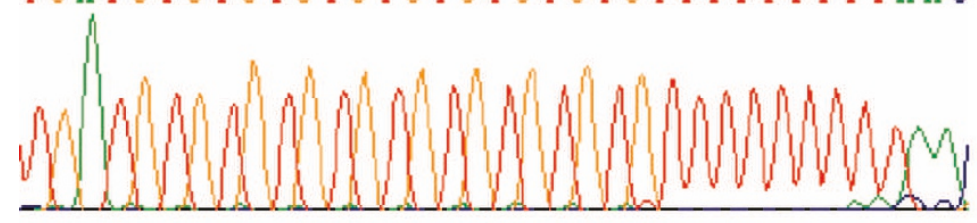

codon 470

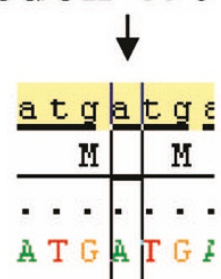

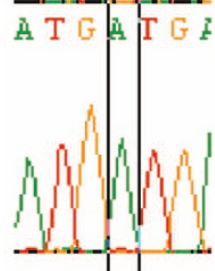

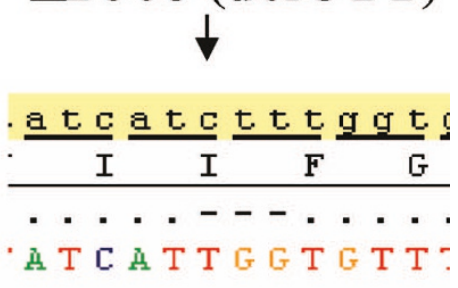

....- - . . . .

ỉ T C A T T G G T T T :

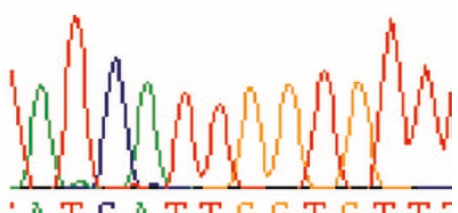

辛 T C $\mathrm{A}$ T T G G T T T ?

$\Delta \mathrm{F508}(\mathrm{delCTT})$

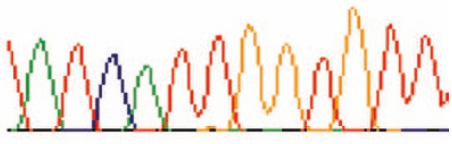

Fig. 1. The $\Delta$ F508 mutation has a 10TG-9T-470M haplotype. Direct sequence analysis for exons 9 and 10 was performed on a genomic DNA sample homozygous for the $\Delta F 508$ mutation. Portions of intron 8 and exon 10 are shown. Results from the forward upper and reverse lower sequencing reactions. 
Sun et al.

a

TG (10/11)

polyT (5/9)

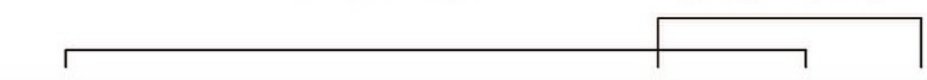

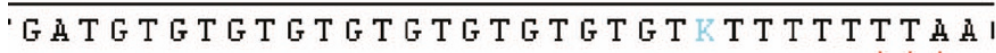

'G

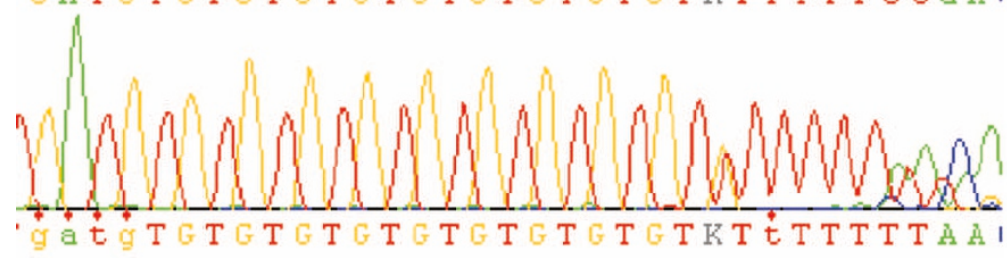

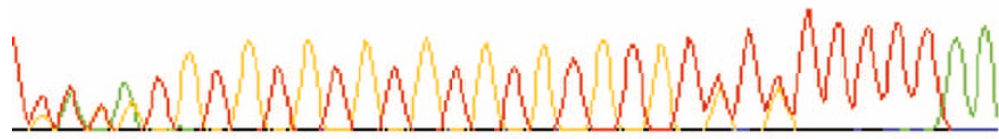

codon $470 \mathrm{M} \quad \Delta \mathrm{F508}(\operatorname{delCTT}) / \mathrm{wt}$

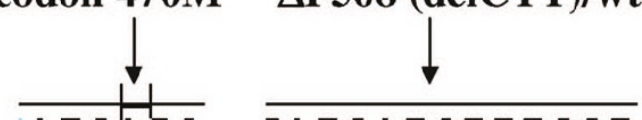

TATCATCTTTGGT

$\Gamma \dot{A} T C \dot{A} T \dot{C} \dot{t} \dot{t} T G G T$
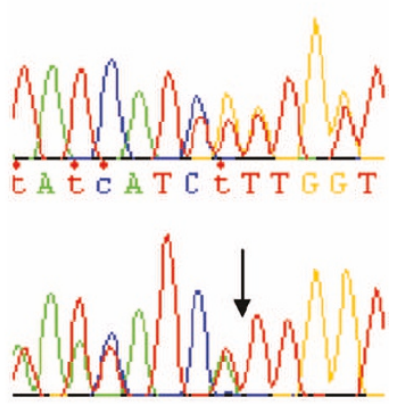

\section{$\Delta$ F508-10TG-9T-470M/wt-11TG-5T-470M}

b TG (10/12) polyT (5/9)

GATGTGTGTGTGTGTGTGTGTGTKTKTTTT TA A

GATGTGTGTGTGTGTGTGTGTETKTKTTTTTA A
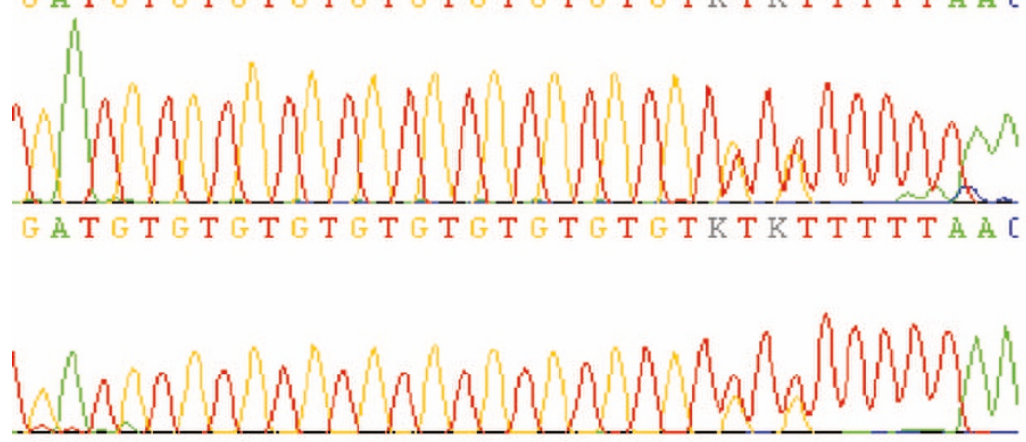

codon $470 \mathrm{M} / \mathrm{V} \quad \Delta \mathrm{F} 508($ delCTT$) / \mathrm{w} 1$
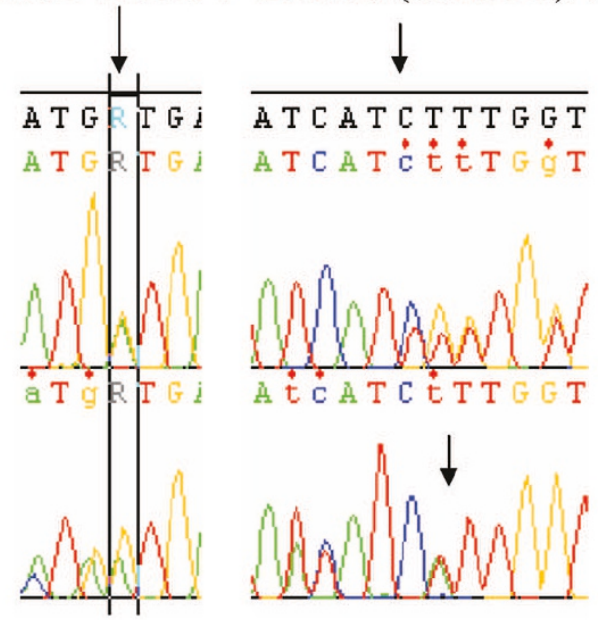

\section{$\Delta$ F508-10TG-9T-470M/wt-12TG-5T-470V}

C TG (10/13)

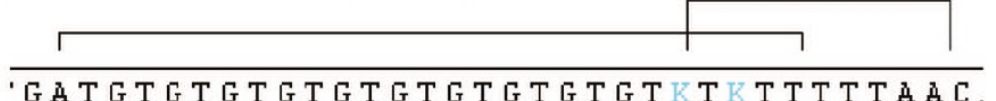

.

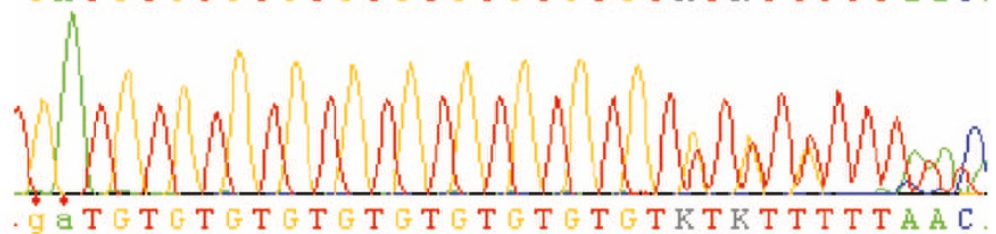

G TGTGTGTGTGTGTGTGTGTGTKTKTTTTTA A

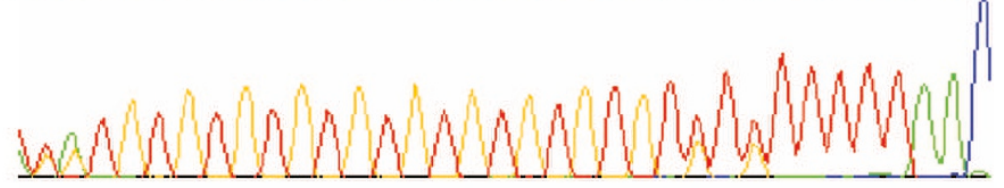

\section{codon $470 \mathrm{M}$}
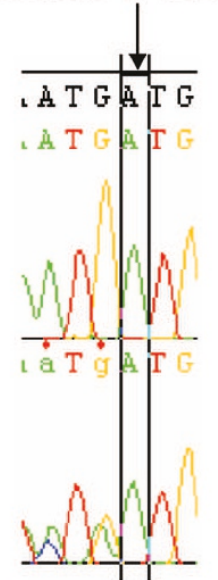

\section{$\Delta$ F508-10TG-9T-470M/wt-13TG-5T-470M}

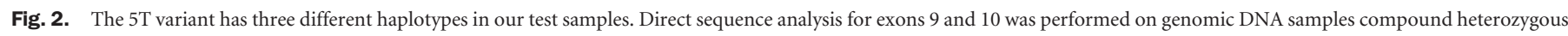

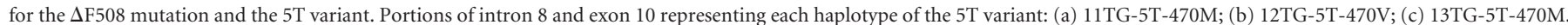
Sequencing reactions from forward (upper) and reverse (lower) directions. 
Table 2

Haplotypes of the $5 \mathrm{~T}$ variant observed in the current study

\begin{tabular}{lc}
\hline 5T Haplotype & Number $(\%)$ \\
\hline 5T-11TG-470M & $41(55 \%)$ \\
5T-12TG-470V & $31(42 \%)$ \\
5T-13TG-470M & $2(3 \%)$ \\
Total & 74
\end{tabular}

\section{Clinical significance of the 5T variant in current study}

Of the 74 specimens included in the 5T haplotype study, only 54 of them have clinical information (even limited) from test requisition forms or from verbal response provided by physicians' offices. Five of the patients (two females and three males) were referred to CF mutation testing by comprehensive gene sequencing as a follow-up of abnormal newborn immunoreactive trypsinogen (IRT) test results. All of them were aged less than 2 months at the time of blood sampling for CF mutation analysis, which might not have allowed sufficient time for CF-related symptoms to manifest. The remaining $49 \mathrm{pa}-$ tients consisted of 34 females and 15 males. This was expected because the majority of specimens referred to our laboratory for $\mathrm{CF}$ analysis are pregnant females seeking carrier screening.

Similar to the observations from our previous study, ${ }^{23}$ penetrance of the $5 \mathrm{~T}$ variant is much lower than expected in females: Only $17.6 \%(6 / 34)$ were symptomatic in the current study (Table 3). In contrast, $67.7 \%$ (10/15) of the males with the same genotype showed atypical CF symptoms. Four of the symptomatic males were affected with CBAVD or azoospermia. However, the common presentations in the symptomatic patients (male or female) occurred in the respiratory system (Table 4): Bronchitis, sinusitis, and asthma/allergy affected six patients. Two infants had abnormal newborn screening (IRT) results with borderline sweat values.

\section{Correlation between the 5T haplotypes and their clinical phenotypes}

As shown in Table 5, among the 34 females, 21 had the 11TG-5T-M470 haplotype, whereas 12 had 12TG-5T-V470 and 1 had the rare 13TG-5T-470M haplotype. However, among the six symptomatic females, five had 12TG-5T-V470 and only one had 11TG-5T-M470. The difference is statistically significant $\left(P=.02, \chi^{2}=4.73\right)$. Therefore, the penetrance is only $4.8 \%(1 / 21)$ for the $5 \mathrm{~T}$ variant with $11 \mathrm{TG}-\mathrm{M} 470$ and $41.7 \%(5 / 12)$ for the $12 \mathrm{TG}-\mathrm{V} 470$ haplotype. Contrary to previous reports ${ }^{16,22,24}$ suggesting high penetrance of the 13TG-

Table 3

Penetrance of the $5 \mathrm{~T}$ variant is low in females in our study population

\begin{tabular}{lccc}
\hline Gender & Symptomatic & Asymptomatic & Penetrance \\
\hline Female & 6 & 28 & $6 / 34(17.6 \%)$ \\
Male & 10 & 5 & $10 / 15(66.7 \%)$ \\
\hline
\end{tabular}

Table 4

Clinical presentations of individuals compound heterozygous for DF508 and 5T

\begin{tabular}{|c|c|c|c|c|}
\hline ID & 5T Haplotype & Gender & Age & Clinical information \\
\hline 1 & $5 \mathrm{~T}-11 \mathrm{TG}-470 \mathrm{M}$ & $\mathrm{F}$ & $44 \mathrm{y}$ & Asthma, recurrent bronchitis \\
\hline 2 & $5 \mathrm{~T}-12 \mathrm{TG}-470 \mathrm{~V}$ & $\mathrm{~F}$ & $27 \mathrm{y}$ & Allergies \\
\hline 3 & $5 \mathrm{~T}-12 \mathrm{TG}-470 \mathrm{~V}$ & $\mathrm{~F}$ & $25 \mathrm{y}$ & Asthma \\
\hline 4 & $5 \mathrm{~T}-12 \mathrm{TG}-470 \mathrm{~V}$ & $\mathrm{~F}$ & $2 \mathrm{wk}$ & $\begin{array}{l}\text { Abnormal newborn screen, } \\
\text { borderline sweat chloride }\end{array}$ \\
\hline 5 & $5 \mathrm{~T}-12 \mathrm{TG}-470 \mathrm{~V}$ & F & $2 \mathrm{mo}$ & $\begin{array}{l}\text { Loose watery stools, nasal } \\
\text { drainage, and mucous } \\
\text { noted in her emesis and } \\
\text { stools }\end{array}$ \\
\hline 6 & $5 \mathrm{~T}-12 \mathrm{TG}-470 \mathrm{~V}$ & $\mathrm{~F}$ & $2 y$ & Borderline sweat \\
\hline 1 & $5 \mathrm{~T}-11 \mathrm{TG}-470 \mathrm{M}$ & M & $5 y$ & $\begin{array}{l}\text { Asthma; non-allergic, } \\
\text { persistent, chronic otitis } \\
\text { media }\end{array}$ \\
\hline 2 & $5 \mathrm{~T}-11 \mathrm{TG}-470 \mathrm{M}$ & M & 36 & $\begin{array}{l}\text { Bronchitis, allergies, sleep } \\
\text { apnea }\end{array}$ \\
\hline 3 & $5 \mathrm{~T}-11 \mathrm{TG}-470 \mathrm{M}$ & M & $5 y$ & $\begin{array}{l}\text { Chronic sinusitis and cough, } \\
\text { failure to thrive }\end{array}$ \\
\hline 4 & $5 \mathrm{~T}-11 \mathrm{TG}-470 \mathrm{M}$ & M & $11 \mathrm{mo}$ & $\begin{array}{l}\text { Atypical symptoms, abnormal } \\
\text { IRT, borderline sweat } \\
\text { chloride }\end{array}$ \\
\hline 5 & $5 \mathrm{~T}-11 \mathrm{TG}-470 \mathrm{M}$ & M & $39 y$ & CBAVD \\
\hline 6 & $5 \mathrm{~T}-12 \mathrm{TG}-470 \mathrm{~V}$ & M & $32 \mathrm{y}$ & CBAVD \\
\hline 7 & $5 \mathrm{~T}-12 \mathrm{TG}-470 \mathrm{~V}$ & M & $19 \mathrm{mo}$ & $\begin{array}{c}\text { Suspected diagnosis of CF, } 2 \\
\text { high sweat chloride value }\end{array}$ \\
\hline 8 & $5 \mathrm{~T}-12 \mathrm{TG}-470 \mathrm{~V}$ & M & $41 \mathrm{y}$ & CBAVD \\
\hline 9 & $5 \mathrm{~T}-12 \mathrm{TG}-470 \mathrm{~V}$ & M & $8 \mathrm{y}$ & $\begin{array}{l}\text { Sweat chloride }(71,61) \text { and } \\
\text { sinus disease }\end{array}$ \\
\hline 10 & $5 \mathrm{~T}-12 \mathrm{TG}-470 \mathrm{~V}$ & M & $26 y$ & $\begin{array}{l}\text { Atypical symptoms, } \\
\text { azoospermia }\end{array}$ \\
\hline
\end{tabular}

IRT, immunoreactive trypsinogen; CBAVD, congenital bilateral absence of vas deferens; $\mathrm{CF}$, cystic fibrosis.

5T-M470 haplotype, the only female (29 years old) in our study with that haplotype was asymptomatic.

Among the male patients, nine had the 11TG-5T-M470 haplotype and six had the 12TG-5T-V470. Similar to our results in females, the 12TG-5T-V470 haplotype had higher penetrance (5/6) than 11TG-5T-M470 (5/9), although the differ-

Table 5

Distribution of $5 \mathrm{~T}$ haplotypes in relation to clinical presentation

11TG-5T-M470 12TG-5T-V470 13TG-5T-M470

Females

Symptomatic

1

Asymptomatic

20

Males

Symptomatic

Asymptomatic

5

4

12TG-5T-V470

13TG-5T-M470

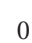

1

$7 \quad 1$


ences did not reach statistical significance, probably because of the low sampling size.

\section{T haplotypes in patients with CBAVD}

Four males with CBAVD or azoospermia as their test indication in our study were added to eight specimens provided by one of the coauthors (A.M.) from a previous study. ${ }^{26,27}$ All were heterozygous positive for the $5 \mathrm{~T}$ variant. The $5 \mathrm{~T}$ variant in 10 of the 12 specimens was found to have the 12TG-5TV470 haplotype. The remaining two specimens had the 11TG5T-M470 haplotype for their 5T alleles. This result is concordant with previous findings that $5 \mathrm{~T}$ variants with adjacent 12 TG repeats were overrepresented in patients with CBAVD when compared with $5 \mathrm{~T}$ with adjacent $11 \mathrm{TG}$ repeats. ${ }^{16}$

\section{DISCUSSION}

The $5 \mathrm{~T}$ variant has been implicated in many atypical CFTRassociated diseases, such as chronic idiopathic pancreatitis, ${ }^{6,7}$ male infertility caused by CBAVD, ${ }^{8,9}$ or respiratory system conditions including chronic rhinosinusitis, ${ }^{10,11}$ bronchiectasis, ${ }^{12}$ and allergic bronchopulmonary aspergillosis. ${ }^{13}$ In vitro studies indicate that chromosomes with the 5T variant have reduced the amount of full-length CFTR gene transcripts because of exon 9 skipping when compared with those with 7T or 9T. ${ }^{17}$ Nevertheless, it is also a frequent variant in the CFTR gene, with an allele frequency of approximately $5 \%$. As shown in our previous study, ${ }^{23}$ the $5 \mathrm{~T}$ variant has an incomplete penetrance since only $3.8 \%$ (7/184) of females and $42.9 \%$ (15/35) of males compound heterozygous for the $\Delta \mathrm{F} 508$ mutation and $5 \mathrm{~T}$ were symptomatic. Compound heterozygosity for the $5 \mathrm{~T}$ variant with a CF mutation is considered to be a major cause of CBAVD. Notably, in a cohort of 92 patients affected with CBAVD, 31 were heterozygous and 1 was homozygous for the $5 \mathrm{~T}$ variant. ${ }^{27}$ In addition, 16 of the $5 \mathrm{~T}$ carriers were also compound heterozygous for the $\Delta$ F508 mutation, making it the most prevalent CFTR genotype in the patients with CBAVD in the study. Nevertheless, the incidence of CBAVD in whites is 1 in 1000 . This comes to only a fraction (22\%) of the individuals compound heterozygous for a CF mutation and the 5T variant $(1 / 222)$ in that population. ${ }^{30}$

Previously, ${ }^{31}$ we described that the I148T CF mutation has a 113-fold increase in prevalence in our screening population when compared with a patient population with CF. An explanation for the differential pathogenicity of this apparently low penetrant mutation is that only $0.6 \%$ of the I148T alleles contains the 3199del6 mutation in exon 17a and 9T in IVS-8. ${ }^{29}$ Similarly, the penetrance of the $5 \mathrm{~T}$ allele can also be caused by different gene background on which the variant arose.

The number of neighboring TG dinucleotide repeats in intron 8 has also been associated with the efficiency of CFTR gene transcription and translation. ${ }^{24}$ On a 7T background, a TG11 allele has a 2.8-fold increase in exon 9 transcripts when compared with a TG10 allele. A TG12 allele has a further increase of up to sixfold in exon 9 skipping. Therefore, the different TGnpoly $\mathrm{T}$ haplotypes in intron 8 have been referred to as "poly- variant" alleles. ${ }^{30}$ The differential combined effect of these polyvariant alleles on exon 9 skipping has been promoted as an explanation for why some patients with $5 \mathrm{~T}$ alleles are symptomatic and others are not. Recent studies showed that the TG12-T5 haplotype is overrepresented in males affected with CBAVD $^{16}$ and chronic pancreatitis, ${ }^{25}$ whereas the TG11-T5 haplotype is more frequently observed in asymptomatic fertile males.

The polyvariant allele of TG12-5T-470V had a higher penetrance than TG11-5T-470M among females in the current study. Similar correlation was also observed in the limited male individuals studied. However, there was a drastically different penetrance value (14.7\% vs. $66.7 \%$ ) when patients are grouped according to gender. It is possible that the $5 \mathrm{~T}$ variant has different phenotypic effects in females versus males. Two independent studies ${ }^{32,33}$ showed that vasal cells had a significantly higher proportion of exon 9-CFTR transcripts when compared with nasal epithelial cells. This differential splicing efficiency between genital tissues and nasal epithelium may partially explain the differential clinical consequence of the $5 \mathrm{~T}$ allele in females versus males. In addition, a recent study showed an enrichment of the $5 \mathrm{~T}$ allele and CF mutations in infertile males but not in infertile females. ${ }^{34}$ The $5 \mathrm{~T}$ variant may indeed be a benign variant in females and a mild mutation in males.

The seemingly higher penetrance of the $5 \mathrm{~T}$ allele in males in the current study may be caused by ascertainment bias. Most females (47/52) included in the study were tested for CF mutations as part of their prenatal screening (for CF carriers). Actually, more than $90 \%$ of the individuals in our CF testing population are females of reproductive age (data not shown). Many males tested may also be part of CF carrier screening, but a much larger fraction are probably tested for diagnostic purposes. The fact that approximately half (7/15) of the male samples included in this study were submitted for CF gene sequencing, a diagnostic assay, confirms this hypothesis. In our previous report based on patients selected from CF screening population, ${ }^{23}$ only 15 of 35 males $(42.9 \%)$ compound heterozygous for the $\Delta \mathrm{F} 508$ mutation and $5 \mathrm{~T}$ were symptomatic. Therefore, the penetrance and haplotype data we observed in males in the current study may have rather limited validity because of ascertainment bias.

In our study, the 13TG-5T haplotype was rare. Previous reports showed that individuals carrying the haplotype were symptomatic with pancreatic-sufficient $\mathrm{CF}^{16,24}$ (in trans to $\Delta$ F508), CBAVD, ${ }^{16,36}$ or asthma ${ }^{22}$ (in trans to R297Q). It was not detected in healthy individuals. In this study, we detected two compound heterozygotes for the 13TG-5T-470M polyvariant allele and the $\Delta$ F508 mutation. Both are females, an asymptomatic 29-year old woman and an asymptomatic 2-month-old infant. The infant was referred to CF gene sequencing because of abnormal IRT results during newborn screening. There is a possibility that she was yet to develop CF-related symptoms, or that she is a CF carrier detected by IRT, a well-described phenomenon.

M470V homozygotes were reported to be overrepresented in individuals affected with chronic rhinosinusitis negative for 
classic CFTR mutations. ${ }^{32}$ Linkage disequilibrium was also found between the 5T allele and the V allele of the M470V polymorphism in CBAVD but not in the general population. ${ }^{37}$ By ignoring the length of TG in female samples, analysis of our data leads to a similar conclusion. The apparently increased penetrance of the $5 \mathrm{~T}$ variant with the $12 \mathrm{TG}-5 \mathrm{~T}-470 \mathrm{~V}$ haplotype could be attributable to the $470 \mathrm{~V}$ allele alone. In support of this hypothesis, the 13TG-5T-470M haplotype, which has the longest TG stretch, seemed to be benign in the current study.

In the current study, not every sample was subjected to comprehensive CF mutation detection by mutation scanning, direct sequencing, or deletion analysis to rule out the presence of additional mutations within the CFTR gene. Comprehensive sequence analysis was performed on only 15 samples. Fortunately, all three haplotypes of the $5 \mathrm{~T}$ variant were observed in these samples. In addition, 10 of the 15 patients who underwent sequencing analysis were symptomatic. Therefore, we do not anticipate that additional CF mutations present in any of the remaining study samples because most of the samples selected from the CF carrier screen (32/38) are asymptomatic.

Our data indicate that the $5 \mathrm{~T}$ variant has limited clinical significance in females when present on an 11TG haplotype. The 5T variant when present with the 12TG-5T-470V haplotype caused symptoms in $40 \%$ of females and $80 \%$ of males in our study. This has important implications in genetic counseling.

\section{References}

1. Collins FS. Cystic fibrosis: molecular biology and therapeutic implications. Science 1992;256:774-779.

2. Zielenski J, Tsui LC. Cystic fibrosis: genotypic and phenotypic variations. Annu Rev Genet 1995;29:777-807.

3. Riordan JR, Rommens JM, Kerem B, Alon N, et al. Identification of the cystic fibrosis gene: cloning and characterization of complementary DNA. Science 1989; 245:1066-1073.

4. Welsh MJ, Ramsey BW, Accurso F, Cutting GR. Cystic fibrosis. In: The Metabolic and Molecular Bases of Inherited Disease, 8th ed. Scriver CR, Beaudet AL, Sly WS, Valle D (eds.). New York: McGraw-Hill 2001:5121-5188.

5. Cystic Fibrosis Genetic Analysis Consortium, 2005. Cystic Fibrosis Mutation Database. http://www.genet.sickkids.on.ca/cftr/. Accessed 1 January 2006.

6. Sharer N, Schwarz M, Malone G, Howarth A, et al. Mutations of the cystic fibrosis gene in patients with chronic pancreatitis. N Engl J Med 1998;339:645-652.

7. Cohn JA, Friedman KJ, Noone PG, Knowles MR, et al. Relation between mutations of the cystic fibrosis gene and idiopathic pancreatitis. N Engl J Med 1998;339:653658.

8. Anguiano A, Oates RD, Amos JA, Dean M, et al. Congenital bilateral absence of the vas deferens. A primarily genital form of cystic fibrosis. JAMA 1992;267:1794-1797.

9. Augarten A, Yahav Y, Kerem BS, Halle D, et al. Congenital bilateral absence of vas deferens in the absence of cystic fibrosis. Lancet 1994;344:1473-1474.

10. Wang X, Moylan B, Leopold DA, Kim J, et al. Mutation in the gene responsible for cystic fibrosis and predisposition to chronic rhinosinusitis in the general population. JAMA 2000;284:1814-1819.

11. Raman V, Clary R, Siegrist KL, Zehnbauer B, et al. Increased prevalence of mutations in the cystic fibrosis transmembrane conductance regulator in children with chronic rhinosinusitis. Pediatrics 2002;109:1-4.

12. Girodon E, Cazeneuve C, Lebargy F, Chinet T, et al. CFTR gene mutations in adults with disseminated bronchiectasis. Eur J Hum Genet 1997;5:149-155.

13. Miller PW, Hamosh A, Macek M Jr, Greenberger PA, et al. Cystic fibrosis transmembrane conductance regulator (CFTR) gene mutations in allergic bronchopulmonary aspergillosis. Am J Hum Genet 1996;59:45-51.
14. Kerem E, Rave-Harel N, Augarten A, Madgar I, et al. A cystic fibrosis transmembrane conductance regulator splice variant with partial penetrance associated with variable cystic fibrosis presentations Am J Respir Crit Care Med 1997;155:1914-1920.

15. Kiesewetter S, Macek M Jr, Davis C, Curristin SM, et al. A mutation in CFTR produces different phenotypes depending on chromosomal background. Nat Genet 1993;5:274-248.

16. Groman JD, Hefferon TW, Casals T, Bassas L, et al. Variation in a repeat sequence determines whether a common variant of the cystic fibrosis transmembrane conductance regulator gene is pathogenic or benign. Am J Hum Genet 2004;74:176-179.

17. Chu CS, Trapnell BC, Curristin S, Cutting GR, et al. Genetic basis of variable exon 9 skipping in cystic fibrosis transmembrane conductance regulator mRNA. Nat Genet 1993;3:151-156.

18. Dork T, Fislage R, Neumann T, Wulf B, et al. Exon 9 of the CFTR gene: splice site haplotypes and cystic fibrosis mutations. Hum Genet 1994;93:67-73.

19. Delaney SJ, Rich DP, Thomson SA, Hargrave MR, et al. Cystic fibrosis transmembrane conductance regulator splice variants are not conserved and fail to produce chloride channels. Nat Genet 1993;4:426-431.

20. Strong TV, Wilkinson DJ, Mansoura MK, Devor DC, et al. Expression of an abundant alternatively spliced form of the cystic fibrosis transmembrane conductance regulator (CFTR) gene is not associated with a cAMP-activated chloride conductance. Hum Mol Genet 1993;2:225-230.

21. Kanavakis E, Tzetis M, Antoniadi T, Pistofidis G, et al. Cystic fibrosis mutation screening in CBAVD patients and men with obstructive azoospermia or severe oligozoospermia. Mol Hum Reprod 1998;4:333-337.

22. Tzetis M, Efthymiadou A, Strofalis S, Psychou P, et al. CFTR gene mutationsincluding three novel nucleotide substitutions-and haplotype background in patients with asthma, disseminated bronchiectasis and chronic obstructive pulmonary disease 2001;108:216-221.

23. Strom CM, Crossley B, Redman J, Buller A, et al. Cystic fibrosis screening: lessons learned from the first 320,000 patients. Genet Med 2004;6:136-140.

24. Cuppens H, Lin W, Jaspers M, Costes B, et al. Polyvariant mutant cystic fibrosis transmembrane conductance regulator genes. The polymorphic (TG)m locus explains the partial penetrance of the T5 polymorphism as a disease mutation. Hum Mol Genet 1998;101:487-496.

25. Fujiki K, Ishiguro H, Ko SB, Mizuno N, et al. Genetic evidence for CFTR dysfunction in Japanese: background for chronic pancreatitis. J Med Genet 2004;41:e55.

26. Hantash F, Milunsky A, Wang Z, Anderson B, et al. A large deletion in the CFTR gene in CBAVD. Genet Med 2006;8:93-95.

27. Wang Z, Milunsky J, Yamin M, Maher T, et al. Analysis by mass spectrometry of 100 cystic fibrosis gene mutations in 92 patients with congenital bilateral absence of the vas deferens. Hum Reprod 2002;17:2066-2072.

28. Strom CM, Huang S, Chen C, Buller A, et al. Extensive sequencing of the cystic fibrosis transmembrane regulator gene: assay validation and unexpected benefits of developing a comprehensive test. Genet Med 2003;5:9-14.

29. Monaghan KG, Highsmith WE, Amos J, Pratt VM, et al. Genotype-phenotype correlation and frequency of the 3199del6 cystic fibrosis mutation among I148T carriers: results from a collaborative study. Genet Med 2004;6:421-425.

30. Claustres M. Molecular pathology of the CFTR locus in male infertility. Reprod Biomed Online 2005;10:14-41.

31. Strom CM, Huang D, Buller A, Redman R, et al. Cystic fibrosis screening using the College panel: platform comparison and lessons learned from the first 20,000 samples. Genet Med 2002;4:289-296.

32. Rave-Harel N, Kerem E, Nissim-Rafinia M, Madjar I, et al. The molecular basis of partial penetrance of splicing mutations in cystic fibrosis. Am J Hum Genet 1997;60: 87-94.

33. Mak V, Jarvi KA, Zielenski J, Durie $\mathrm{P}$, et al. Higher proportion of intact exon 9 CFTR mRNA in nasal epithelium compared with vas deferens. Hum Mol Genet 1997;6: 2099-2107.

34. Morea A, Cameran M, Rebuffi AG, Marzenta D, et al. Gender-sensitive association of CFTR gene mutations and 5T allele emerging from a large survey on infertility. Mol Hum Reprod 2005;11:607-614.

35. Wang X, Moylan B, Leopold DA, Kim J, et al. Mutation in the gene responsible for cystic fibrosis and predisposition to chronic rhinosinusitis in the general population. JAMA 2000;284:1814-1819.

36. Costes B, Girodon E, Ghanem N, Flori E, et al. Frequent occurrence of the CFTR intron 8 (TG)n5T allele in men with congenital bilateral absence of the vas deferens. Eur J Hum Genet 1995;3:285-293.

37. de Meeus A, Guittard C, Desgeorges M, Carles S, et al. Linkage disequilibrium between the M470V variant and the IVS8 polyT alleles of the CFTR gene in CBAVD. J Med Genet 1998;35:594-596. 